Brit. Heart F., 1968, 30, 402.

\title{
Left Heart Catheterization by Direct Ventricular Puncture
}

\author{
T. SEMPLE, J. B. McGUINNESS, AND H. GARDNER \\ From the Cardiac and Radiology Departments, Victoria Infirmary, Glasgow S.2
}

Attempts at complete catheterization of the left heart by transseptal and retrograde routes are not always completely informative nor entirely free from complications (Crowley and Parkin, 1956; KavanaghGray and Drake, 1958; Aldridge, 1964; Samet, Bernstein, and Levine, 1965; Christiansen and Wennevold, 1966), and further alternative means of obtaining haemodynamic information from the left side of the heart seem invaluable.

Levy and Lillehei (1964) described a method of obtaining pressure gradient readings across the aortic and mitral valves by the use of a needle-type short catheter introduced to the ventricle directly through the chest wall at the cardiac apex. We have modified this technique by using a simpler needletype teflon catheter, and, in a proportion of patients, by introducing a larger catheter for ventriculography and aortography by a variation of the Seldinger technique. After evaluating it in dogs we have now employed the method in a pilot study of 55 patients.

\section{TeChNIQUE}

The patient, premedicated with pethidine, lies supine and the apex beat is located by palpation. The site and direction of entry at the apex is determined by screening a forceps tip or needle. This site is anaesthetized down to the pericardium, and after a minute or two, a small skin puncture hole is made with the tip of a scalpel.

A 19-gauge needle, $20 \mathrm{~cm}$. (8 in.) long, over which an 18-gauge teflon catheter fits snugly, is connected by a length of tubing to the pressure transducer. The catheter with needle trocar is now advanced through the skin and subcutaneous tissues, pointing in the long axis of the heart towards the second right chondrosternal junction until the tip is seen and felt to touch the apex of the heart. It is then inserted rapidly through the ventricular wall, entry into the left ventricle being confirmed immediately by the appearance of a left ventricular pressure tracing on the oscilloscope. When this is observed the teflon catheter is advanced an inch or so beyond the

Received September 25, 1967. needle and the needle withdrawn, leaving the teflon catheter alone in the left ventricle. This is reconnected to the polythene tubing and the system flushed out. In most cases, further gentle advancement of the soft tefion catheter takes it into the aorta along with the blood flow. If this does not occur a Seldinger guide wire is introduced and this usually passes easily into the aorta, the tefion catheter following. In a similar way left atrial catheterization may be attempted through the mitral valve. Pressures are recorded in each site and pressure gradients recorded across the mitral and aortic valves.

In cases where angiography is performed, a Seldinger wire is introduced, the teflon catheter is removed, and a No. 6 or 7 Gensini catheter is passed into the heart. When the catheter tip is placed satisfactorily in the appropriate chamber, angiocardiography is carried out in the usual way. If attempted catheterization of the left atrium has been unsuccessful, a further attempt can be made using the stiffer catheter. In the earliest patients studied, the catheter was stitched to the chest wall, since Levy and Lillehei had noted recoil of the catheter at the time of pressure injection, but later we found this to be unnecessary with the Gensini catheter.

After the procedures have been completed the catheter is removed from the heart and pressure maintained over the puncture for two or three minutes. The whole undertaking usually takes about 20 to 30 minutes, but if no angiography is required, pressure records may be obtained in a matter of 10 to 15 minutes.

\section{SUBJECTS}

Studies were made on 22 dogs in an attempt to evaluate the method. Special interest was taken in the amount of bleeding into the pericardium and pleura, ease of entry into the desired areas, damage to the lung, and the incidence of serious arrhythmias.

Eighteen dogs were divided into three groups of six. The first group had one clean ventricular puncture and the full procedure carried out using a No. 6 Gensini catheter as described above. The second group had three cardiac punctures and then the full procedure carried out once using a No. 6 Gensini catheter, since it was felt that the effect of several attempts at this procedure should be known. The third group had one cardiac 
TABLE I

HAEMORRHAGIC EFFECTS OF VENTRICULAR PUNCTURE CATHETERIZATION IN 18 DOGS

\begin{tabular}{c|c|c|c|c||c|c|c}
\hline & \multicolumn{3}{|c||}{ Blood in pericardium (ml.) } & \multicolumn{3}{c}{ Blood in pleura (ml.). } \\
\cline { 2 - 7 } & $<5$ & $5-10$ & $>10$ & Tamponade & $<5$ & $5-10$ & $>10$ \\
\hline Group A: 1 puncture, & 6 & 0 & 0 & 0 & 6 & 0 & 0 \\
$\begin{array}{c}\text { Gensini catheter } \\
\text { Group B: 3 punctures, }\end{array}$ & 2 & 1 & 0 & 3 & 3 & 3 & 0 \\
$\begin{array}{c}\text { Gensini catheter } \\
\text { Group C: 1 puncture, } \\
\text { larger Kifa catheter }\end{array}$ & 1 & 3 & 2 & 0 & 3 & 2 & 1 \\
\hline
\end{tabular}

puncture and procedure attempted using a grey Kifa catheter in order to assess whether or not it would be possible to use a much larger catheter with safety. Following the study the dogs were immediately killed and the heart and chest examined. The amount of blood free in the pericardium and the pleural sac was measured and the presence or absence of pulmonary damage noted.

In order to investigate at which stage bleeding occurred four dogs were anaesthetized, the chest and pericardium were opened, and the whole procedure carried out under direct vision using a No. 6 Gensini catheter in each case.

Studies were made on 55 patients, 51 of whom had or were suspected of having rheumatic valvular disease; 2 had atheroma and 2 had cardiomyopathy. In 45 instances, the complete procedure with angiography using a Gensini catheter was carried out, while in 10 patients pressure records using only the fine teflon catheter were obtained.

\section{RESULTS}

Dogs. The results in the three groups are shown in Table I. Group A seems to have fared best and it seems from these observations that, as one might expect, the fewer the punctures and the smaller the catheter the better for the patient. No serious arrhythmias occurred in these dogs. The aorta was entered easily in each instance and the left atrium entered in 8 of the 18 procedures $(45 \%)$. In 8 of the dogs the needle puncture was seen on the anterior border of the left lung.

The open chest procedure helped to shed light on the time of occurrence of the haemorrhage. With the catheter and needle trocar through the ventricular wall no bleeding occurred even when the needle was put through a wide range of movement, much more than would be expected in a normal left ventricular puncture. When the teflon catheter was in alone, following withdrawal of the needle, no bleeding occurred. The guide wire was then inserted and the teflon catheter removed over it; at this stage a fairly constant ooze of blood was seen to occur past the guide wire, and it was our impression that this wire was acting almost as a saw and could damage the myocardium if left in long enough. As would be expected, when the Gensini catheter was introduced over the wire the bleeding stopped, but when this catheter was removed a small hole could be seen in the myocardium through which blood emerged in jets for four or five beats before it closed, being followed by a very slight ooze for a minute or two.

Patients. The left ventricle was entered in all 55 patients and an aortic valve gradient was obtained in all but 2 of these. In this respect, even severe aortic stenosis presented no problem. The left atrium was entered in 21 instances. Midway through the series it became apparent that it was not difficult to reach the right ventricle by continuing to advance the needle and catheter through the septum from the left ventricle, but this was only performed in 7 patients.

In 8 patients known to have aortic stenosis alone the gradient was measured by the small teflon catheter only and the Gensini catheter was not inserted. In 45 patients the larger catheter was used in addition, in order to obtain an angiogram in the left ventricle.

In all, 28 patients experienced pain which was either praecordial, pleuritic, or both; 17 had retrosternal pain and in 14 it was typically pleuritic. The time of onset of this pain and severity are shown in Table II. In only one patient was pain severe enough to cause the procedure to be cut short, and this was in a patient in whom the guide wire inadvertently slipped into the pericardial space. It is interesting to note that as a general rule retrosternal pain tended to be more severe and to come on earlier

TABLE II

TIME OF ONSET AND TYPE OF PAIN IN 28 PATIENTS WHO EXPERIENCED THIS (SEVERAL HAD BOTH TYPES OF PAIN)

\begin{tabular}{ll|c|c}
\hline & & Praecordial (17) & Pleuritic (12) \\
\cline { 2 - 3 } During procedure.. &. & 13 & 7 \\
After procedure &. & 4 & 7 \\
\hline
\end{tabular}


in the procedure, whereas pleuritic pain was usually less severe and came on towards the end or even after the procedure had been terminated.

Blood pressure fell from its original level in 6 patients. In only one was this accompanied by pallor and sinus tachycardia, and this was in the patient mentioned above in whom the guide wire entered the pericardium. Routine chest $x$-ray examination was carried out a few hours after the procedure and a week later. Six patients showed changes, there being two small pleural effusions, two small pneumothoraces, and two instances of intramural injection of contrast medium. Changes in clinical signs were observed in 6 patients; 2 had transient pleuro-pericardial friction and one had transient pleural friction. One was shocked, as mentioned above, and recovered in a few hours with no treatment. In 2 patients there was a mild hemiplegia which came on shortly after the procedure: this passed off in a few days and was thought to be related more to the angiocardiogram than to the actual procedure of left ventricular catheterization. These 2 patients were the 2 who suffered from primary arteriosclerotic disease. The patient with pleural friction was one of the 2 who developed pleural effusion subsequently, and this patient was readmitted one month later with a recurrence of pleuritic pain and a recurrence of effusion. At this time the erythrocyte sedimentation rate was raised, there was a slight pyrexia, and the picture presented was that of the post-pericardiotomy syndrome.

One patient died during the procedure: this was a girl of 14 years of age who had had severe retrosternal pain on exertion for some months and had been admitted to hospital three months previously with dyspnoea, haemoptysis, and a clinical picture suggestive of pulmonary infarction. She had a harsh ejection systolic murmur at the base of the heart and the electrocardiogram showed $T$ wave inversion in leads V4-V6, with increased voltage of the QRS complexes. It was felt that she had possibly an obstructive cardiomyopathy, and left heart catheterization was undertaken. The initial procedure was entirely without incident: the aorta was easily entered and catheterized. When the catheter entered the aorta it followed an unusual course, turning on itself and heading towards the diaphragm. It was felt that it had entered a coronary artery and a hand injection of contrast medium was made. Immediately the patient developed ventricular fibrillation and despite resuscitative measures this could not be reversed. Necropsy confirmed that the patient suffered from cardiomyopathy. We felt this incident to be the result of the injection of contrast medium into the coronary artery rather than due to the trauma of the catheterization procedure.
During catheterization itself serious arrhythmia was not a problem. Most patients had one or two ventricular extrasystoles and one had nodal tachycardia as the catheters were introduced, while one had a run of nodal tachycardia and another a short burst of ventricular tachycardia shortly after the injection of contrast medium. These were all of such short duration that no treatment was necessary.

There were remarkably few technical problems in this series. In one instance the guide wire entered the pericardium, as already mentioned, causing pain severe enough to cause termination of the procedure. In another case the small radio-translucent teflon catheter looped outside the heart probably in the pericardium. This was probably due to failure to advance the teflon catheter sufficiently before withdrawing the rigid needle. Whatever the reason, the guide wire could not be passed through the looped catheter despite the fact that a free ventricular tracing was being obtained and blood could be removed via the catheter. These two cases account for the two failures to enter the aorta. In yet another patient the Gensini catheter would not enter the heart over the guide wire and the procedure had to be abandoned at this stage.

One patient in the series died seven days after the procedure: he had mitral regurgitation and had been in failure for some time before the investigation, having been treated in hospital for three weeks in an attempt to improve his condition. He was known to be a poor risk before the procedure, but it was felt that operation was essential, and that before this more information should be obtained about the mitral valve. Six days after the procedure he developed a tachycardia, the blood pressure began to fall, and he became dyspnoeic. His condition deteriorated rapidly and he died the next day. Necropsy revealed a large pulmonary embolism which had given rise to infarction at the base of the left lung. Examination of the heart showed a little pericarditis over the anterior surface but no evidence of the preceding left ventricular puncture.

\section{Discussion}

Direct approach to the left ventricle across the chest wall is not a new conception. It was first described in 1933 when Reboul and Racine obtained ventriculograms in this way in dogs. Its value as a practical method of obtaining information from the human left ventricle was predicted some years later by Buchbinder and Katz (1949) after they had inserted a needle through the cardiac apex of a moribund patient. Since then several reports of this direct approach have appeared, using either apical or sub-xiphoid puncture (Smith et al., 1954; Smith, 
Cregg, and Klassen, 1956; Cregg et al., 1955; Fleming and Gibson, 1957; Fleming et al., 1958; Björk et al., 1961). Using a metal needle the incidence of serious complications in these series was small but significant. The risk of laceration of a coronary artery and/or significant intrapericardial haemorrhage was mentioned in each, and this, together with an emotional barrier to piercing the heart directly by a needle, seems to have given rise to a certain aversion to the technique, possibly somewhat undeserved in the light of published evidence. It should also be remembered that these results were reported in the days when a metal needle alone was used and was often left in situ for upwards of 10 to 15 minutes.

The series of 122 cases using the short pliable catheter needle reported by Levy and Lillehei (1964) was remarkably free from complications, there being only one case of significant haemopericardium. Their one death was due to an intramural injection of contrast medium in a child with a complicated congenital lesion, an accident that can occur with any technique of left ventricular angiography. Accordingly we were encouraged to explore the method in animals. We found the experience gained in the dogs invaluable in that it showed clearly that this even more flexible needle, through the ventricular wall for a very short space of time, was not associated with significant blood loss into the pericardial space. It also clearly demonstrated that the fewer the punctures and the smaller the catheter used, the less blood might be expected to escape. In addition, we realized the necessity for speed when changing catheters over the guide wire. For this reason we have always employed and would recommend two experienced operators for the performance of this additional ventriculography procedure in patients.

The technical problems in our series of 55 patients have been remarkably few. It is very important that, when the teflon sheath with its needle trocar has reached the cavity of the left ventricle, the first movement before withdrawing the needle should be advancement of the teflon catheter an inch or two. This often takes it up the outflow stream into the aorta, but, more important, it will prevent the soft catheter from entering the pericardium and looping outside the heart as happened in two of our early patients.

The most common clinical complication was pain, but in one patient only was this sufficiently severe to cause us to abandon the procedure. The pain seems undoubtedly related to extravasation of blood; thus in 8 patients in whom the small teflon catheter alone was used to obtain an aortic valve gradient, no pain whatsoever occurred. Excluding pain, all other complications were confined to 12 patients, 5 of whom suffered effects of contrast-medium injection rather than of the technique employed; thus 2 patients had transient hemiplegia, one had nodal tachycardia, and 2 suffered a small intramural injection of contrast medium, in one of whom it induced a short burst of nodal tachycardia. Like others (Björk et al., 1961), we found that cardiac arrhythmias were not a real problem, usually consisting, if present at all, of a few ventricular extrasystoles as the needle was introduced. Our one example of ventricular fibrillation was an unfortunate but well-recognized complication of selective coronary angiography.

From this series and that of Levy and Lillehei (1964) we feel justified in saying that this method of entry to the left heart for pressure readings is a safe technique. It has the advantage of providing certain access to the left ventricle and, even in the most severe aortic stenosis, easy access to the aorta. We obtained entry into the left atrium in 38 per cent of the cases, but this figure would probably have been higher had we been more persistent. In most instances we were content to enter the ventricle and aorta, and, if indicated, assess the systolic competence of the mitral valve by ventriculography, interpreting the results in the way described by Rees, Jefferson, and Harris (1965). A further feature which we found attractive was the short duration of the procedure; with some experience, the time from cardiac puncture through pressure readings to angiography took some 15 to 30 minutes. In addition, the semirigid metal needle was in the heart for less than 5 seconds, only the completely flexible teflon catheter remaining in situ during the investigation. We believe this to be the most important aspect of the technique, in that most of the complications previously reported with ventricular puncture were in all probability related to myocardial damage produced by respiratory and cardiac movements of the rigid metal needle in situ, sometimes for an appreciable number of minutes. We would agree with Levy and Lillehei that this approach is extremely well suited to the investigation of severe aortic stenosis. It is a useful alternative method of performing left ventricular angiography for the assessment of mitral regurgitation, particularly if the aortic valve also requires study. It must be conceded however that angiography, requiring the insertion of a conventional thick-wall, slightly stiffer catheter, significantly increases the morbidity. The technique may be employed for access to the left ventricle when the retrograde method fails or is likely to fail because of coarctation or severe atheroma, and may even be used in these circumstances for root or arch aortography.

It is not suggested that the method should be used to the exclusion of present standard techniques but 
we feel that, with this flexible apparatus, present reluctance to perform direct left ventricular puncture is hardly justified, especially when used to acquire important information which cannot be obtained by the more conventional methods.

\section{SUMMARY}

A comparatively safe method of left ventricular puncture using a $20 \mathrm{~cm}$. (8 in.) 18-gauge teflon catheter has been evaluated in dogs. It was later used in 55 patients most of whom had rheumatic valvular disease. It can be used as an alternative to left heart catheterization and for measuring the systolic aortic valve gradient. By adding the Seldinger technique and accepting a slight additional morbidity, the technique described can be modified to produce satisfactory left ventriculograms.

\section{REFERENCES}

Aldridge, H. E. (1964). Transseptal left heart catheterisation without needle puncture of the interatrial septum. Amer. F. Cardiol., 13, 239.

Björk, V. O., Cullhed, I., Hallén, A., Lodin, H., and Malers E. (1961). Sequelae of left ventricular puncture with angiocardiography. Circulation, 24, 204.

Buchbinder, W. C., and Katz, L. N. (1949). Intraventricular pressure curves of the human heart obtained by direct transthoracic puncture. Proc. Soc. exp. Biol. (N.Y.), 71, 673.
Christiansen, I., and Wennevold, A. (1966). Complications in 1,056 investigations of the left side of the heart. Amer. Heart F., 71, 601.

Cregg, H. A., Smith, P. W., Wilson, C. W., and Bull, J. W. (1955). Cardioangiography. Radiology, 65, 368.

Crowley, W. P., and Parkin, T. W. (1956). Experience with and sequelae of catheterisation of the left side of the heart via the percutaneous route. Proc. Mayo Clin., 31, 115.

Fleming, H. A., Hancock, E. W., Milstein, B. B., and Ross, D. N. (1958). Percutaneous left ventricular puncture with catheterisation of the aorta. Thorax, $13,97$.

Fleming, P., and Gibson, R. (1957). Percutaneous left ventricular puncture in the assessment of aortic stenosis. Thorax, 12, 37.

Kavanagh-Gray, D., and Drake, E. H. (1958). Complications of left heart catheterisations using the right transthoracic approach. Amer. Heart F., 56, 143.

Levy, M. J., and Lillehei, C. W. (1964). Percutaneous direct cardiac catheterisation. A new method with results in 122 patients. New Engl. F. Med., 271, 273.

Reboul, H., and Racine, M. (1933). Ventriculographie cardiaque expérimentale. Presse méd., 37, 763.

Rees, R. S. O., Jefferson, K. E., and Harris, A. M. (1965). Cineangiography of the mitral valve. Brit. Heart $\mathcal{F}$., 27, 498.

Samet, P., Bernstein, W. H., and Levine, S. (1965). Transseptal left heart catheterisation. An analysis of 390 studies. Dis. Chest, 48, 160.

Smith, P.W., Cregg, H. A., and Klassen, K. P. (1956). Diagnosis of mitral regurgitation by cardioangiography. Circulation, 14, 847.

—, Wilson, C. W., Cregg, H. A., and Klassen, K. P. (1954). Cardioangiography. F. thorac. Surg., 28, 273. 